\title{
Determinação das curvas de secagem das sementes de andiroba em secador solar
}

Andreza P. Mendonça ${ }^{1}$, Paulo de T. B. Sampaio ${ }^{2}$, Francisco de A. C. Almeida ${ }^{3}$, Raissa F. Ferreira ${ }^{4}$ \& Juliene M. Novais ${ }^{5}$

\section{Palavras-chave:}

modelos matemáticos

Carapa surinamensis Miq

Carapa guianensis Aubl

\begin{abstract}
R E S U M O
Comumente, o óleo de andiroba é extraído na Amazônia pelo método tradicional ou ainda por prensa. A eficiência na extração está relacionada ao aquecimento e ao teor de água das sementes. Desta forma, a determinação de um modelo de secagem que represente satisfatoriamente os dados experimentais é de suma importância para minimizar as alterações promovidas pelo processo obtendo-se, consequentemente, produto de qualidade. O objetivo do trabalho foi descrever a cinética de secagem das sementes de andiroba, tal como ajustar os modelos matemáticos aos dados experimentais usando-se secador solar. Foi utilizado, como critério do ajuste dos modelos matemáticos, o coeficiente de determinação, a magnitude do erro médio relativo e o desvio-padrão da estimativa. A secagem em menor tempo (14 dias) da Carapa surinamensis para atingir o teor de água de equilíbrio $(12,28 \%)$ se deve, possivelmente, ao menor tamanho das sementes e à maior quantidade de óleo em relação à Carapa guianensis. O modelo Logarítmico e o de Midilli et al. foram os que melhor se ajustaram aos dados experimentais para as sementes das duas espécies de andiroba.
\end{abstract}

\section{Key words:}

mathematical models

Carapa surinamensis Miq

Carapa guianensis Aubl

\section{Determination of drying curves of crabwood in solar dryer}

\section{A B S T R A C T}

The oil of crabwood is commonly extracted in Amazon by traditional or by pressing method. The extraction efficiency is related to the heating and water content of the seeds. Thus, the determination of a drying model that represent satisfactorily the experimental data is of paramount importance to minimize the changes introduced by the process, consequently obtaining a quality product. The objective of this study was to describe the drying kinetics of seeds of crabwood as well as adjust the mathematical models to the experimental data, using solar dryer. The coefficient of determination, the magnitude of the mean relative error and standard deviation of the estimate was used as the criterion of fit of mathematical models. Drying in a shorter period of time (14 days) of Carapa surinamensis to reach the water equilibrium content $(12.28 \%)$ may be attributed to the smaller size of the seeds and the greater amount of oil compared to Carapa guianensis. Logarithmic and Midilli et al. were the model that best fitted the experimental data for seeds of both species of Andiroba.
\end{abstract}




\section{INTRODUÇÃo}

A produção de óleos vegetais nas comunidades rurais da Amazônia tem garantido a conservação e a diversificação da floresta e assegurado renda às famílias. Entre as espécies florestais com potencial de contribuir para o desenvolvimento econômico da região se encontra a andiroba.

O gênero Carapa, pertence à família das Meliaceae é uma das três espécies botânicas Carapa surinamensis Miq, Carapa vasquezii Kenfack e Carapa guianensis Aubl, com o mesmo nome vulgar andiroba. O óleo de andiroba é bastante utilizado na indústria de cosmético e fitoterápico podendo ainda ser utilizado para produção de biodiesel (Mendonça \& Ferraz, 2007; Kenfack, 2011). A característica físico-química do óleo é o fator limitante para seu uso necessitando, portanto, de procedimentos adequados de secagem e extração do óleo que assegurem sua qualidade.

A secagem é um dos procedimentos importantes da extração do óleo. Neste processo, a temperatura pode afetar as propriedades do óleo (Silva et al., 2007). Desta forma, a determinação do efeito da temperatura e a obtenção de um modelo de secagem que represente satisfatoriamente os dados experimentais, são de suma importância para minimizar as alterações promovidas pelo processo obtendo-se, consequentemente, produtos de qualidade.

$\mathrm{O}$ estudo de sistemas de secagem, seu dimensionamento, otimização e a determinação da viabilidade de sua aplicação comercial, podem ser feitos por meio de simulação matemática cujo princípio se fundamenta na secagem de camadas delgadas do produto, utilizando um modelo matemático que representa satisfatoriamente a perda de água (Resende et al., 2007).

A secagem de sementes pode ser descrita pelos métodos teóricos, semiteóricos e empíricos. Nos métodos teóricos consideram-se as condições externas sob as quais a operação ocorre como também os mecanismos internos de transferência de energia e massa e seus efeitos. Entre os modelos teóricos pode-se citar: fluxo hidrodinâmico e as difusões: líquida, capilar, superficial, de vapor e térmica (Almeida et al., 2006).

Os modelos semiteóricos se baseiam na Lei de Newton para resfriamento aplicada para transferência de massa presumindo-se que as condições sejam isotérmicas e que a resistência à transferência de água se restrinja apenas à superfície do produto (Almeida et al., 2006). Entre os modelos semiteóricos estão o modelo de Henderson \& Padis (1961) e o de Page (1949).

O método empírico consiste em formar grupos físicos adimensionais que podem ser facilmente investigados por experimentos de laboratórios e se baseia nas condições externas, como temperatura, razão da mistura e velocidade do ar de secagem. Dentre os modelos, pode-se citar: Thompson et al. (1968) e o de Wang \& Sing (1978).

Na Amazônia é comum a secagem de sementes oleaginosas em terreiro de concreto usando ventilação natural cujo controle do teor de água é realizado de maneira empírica nas comunidades rurais; contudo, há pouca ou nenhuma informação na literatura sobre cinética de secagem ou ainda o efeito do manejo das sementes sobre a qualidade do óleo extraível.
Com base no exposto objetivou-se, neste trabalho, descrever a cinética de secagem das duas espécies de andiroba usando secador solar, além de ajustar diferentes modelos matemáticos aos dados experimentais.

\section{Material e Métodos}

As sementes de andiroba (C. surinamensis Miq e C. guianensis Aubl.) foram coletadas em plantios em propriedade particular localizada na linha 04 , na zona rural, em Ouro Preto d'Oeste, Rondônia (latitude: $10^{\circ} 47^{\prime} 22^{\prime \prime} \mathrm{S}$ e longitude: $62^{\circ}$ $10^{\prime} 22^{\prime}$ W) no período de março a maio. Após a coleta suas sementes foram beneficiadas e mantidas imersas em água, durante $24 \mathrm{~h}$ a fim de matar, por afogamento, a Hypsipyla e homogeneizar o teor de água das sementes.

As sementes das duas espécies de andiroba $(n=150)$ tiveram o comprimento, a largura e a espessura medidos com auxílio de um paquímetro digital em $\mathrm{mm}$ e o peso por meio de uma balança semianalítica com precisão de $0,01 \mathrm{~g}$.

O teor de água, tanto inicial como final, foi determinado pelo método padrão de estufa a $105 \pm 3{ }^{\circ} \mathrm{C}$, por $24 \mathrm{~h}$, utilizandose quatro repetições de acordo com as Regras de Análise de Sementes (Brasil, 2009). A percentagem de teor de água foi calculada em base úmida aplicando-se a Eq. 1, sendo o resultado final expresso pela média aritmética das subamostras.

$$
\mathrm{Xi}=\frac{w i-w s}{w i}
$$

em que:

$\mathrm{Xi} \quad$ - teor de água

wi - massa úmida

ws - massa seca

A cinética de secagem das duas espécies de andiroba foi realizada em secador solar usando-se sementes inteiras e com casca. As sementes foram colocadas em triplicata em cesta de arame vazado formando uma amostra em monocamada. $\mathrm{O}$ acompanhamento da secagem ocorreu por meio de quatro repetições sendo as pesagens das sementes realizadas em balança semianalítica com precisão de $0,01 \mathrm{~g}$ até se obter peso constante, equivale a determinar o teor de água de equilíbrio para as específicas condições termodinâmicas do ar de secagem.

O secador solar possui as dimensões de 2,5 $\mathrm{m}$ de altura por $4 \mathrm{~m}$ de largura coberto por lona transparente anti-UV de 150 mica, formado por 9 prateleiras com fundo vazado de arame galvanizado possibilitando a circulação de ar entre as sementes. O piso do secador é de concreto. No interior do secador foi instalado um exaustor. $\mathrm{O}$ registro da temperatura e umidade no interior do secador foi realizado por meio de um termohigrômetro digital diariamente (Figura 1).

As curvas de secagem foram obtidas pela conversão dos dados referentes à perda de água no parâmetro adimensional razão de teor de água (RX). A razão de teor de água é essencial para descrever diferentes modelos de secagem em camada delgada. A cada tempo de secagem um teor de água é correlacionado com o teor de água inicial e o teor de água de equilíbrio, para condições especificas de secagem. Desta forma, 


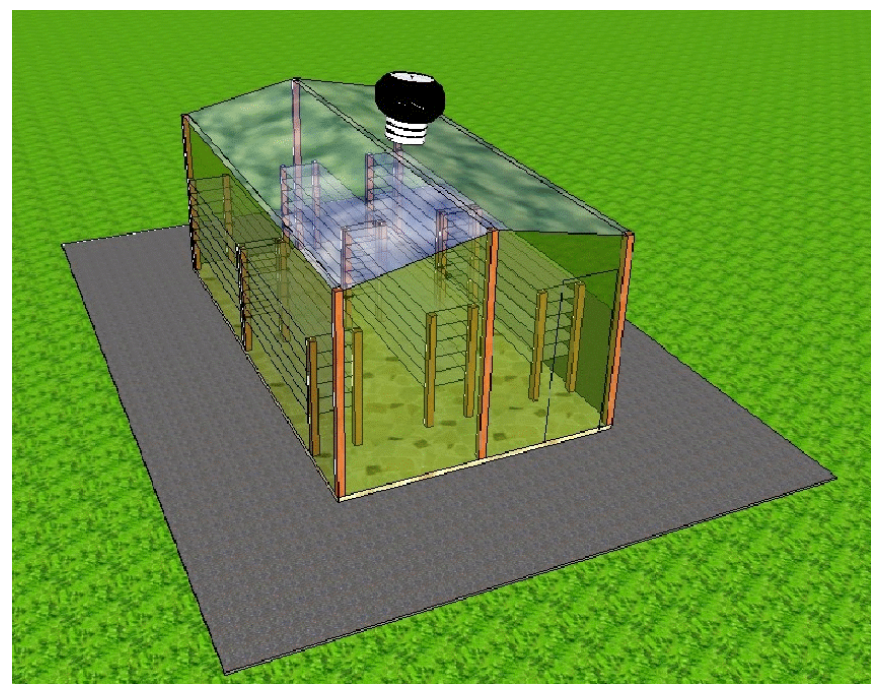

Figura 1. Desenho esquemático do secador solar usado na secagem das sementes de andiroba (C. surinamensis Miq. e C. guianensis Aubl.)

em todos os modelos matemáticos testados os valores de razão de umidade em função do tempo de secagem foram ajustados aos modelos, descrevendo a cinética de secagem.

Os ajustes matemáticos das curvas de cinética de secagem foram realizados por meio dos modelos descritos na Tabela 1 utilizando-se o programa computacional Statistic, versão $7.0^{\circ}$ por meio de análise de regressão não linear, pelo método Quasi-Newton.

Tabela 1. Modelos matemáticos utilizados para predizer o fenômeno de secagem de sementes inteiras e com casca da andiroba (C. surinamensis Miq e C. guianensis Aubl.)

\begin{tabular}{ll}
\hline $\begin{array}{c}\text { Designação dos modelos } \\
\text { matemáticos }\end{array}$ & \multicolumn{1}{c}{ Equação } \\
Aproximação da difusão & $\mathrm{RX}=\mathrm{a} \cdot \exp (-\mathrm{kt})+(1-\mathrm{a}) \cdot \exp (-\mathrm{kbt})$ \\
Logarítmico & $\mathrm{RX}=\mathrm{a} \cdot \exp (-\mathrm{kt})+\mathrm{c}$ \\
Midilli et al. (2002) & $\mathrm{RX}=\mathrm{a} \cdot \exp \left(-\mathrm{kt} \mathrm{t}^{\mathrm{n}}\right)+\mathrm{bt}$ \\
Newton & $\mathrm{RX}=\exp (-\mathrm{kt})$ \\
Page (1949) & $\left.\mathrm{RX}=\exp (-\mathrm{kt})^{\mathrm{n}}\right)$ \\
Thompson et al. (1968) & $\mathrm{RX}=\exp \left\{\left[-\mathrm{a}\left(\mathrm{a}^{2}+4 \mathrm{bt}\right)^{0,5}\right] / 2 \mathrm{~b}\right\}$ \\
\hline
\end{tabular}

$\mathrm{k}$ - Constante de secagem; a, b, $\mathrm{n}$ - Constante do modelo; $\mathrm{t}$ - Tempo, min

Os critérios usados para determinação do melhor ajuste dos modelos aos dados experimentais foram o coeficiente de determinação $\left(\mathrm{R}^{2}\right)$, a magnitude do erro médio relativo (P) e do desvio-padrão da estimativa (SE) e a tendência de distribuição dos resíduos (aleatória ou tendenciosa). O resíduo é a diferença entre o valor observado experimentalmente pelo valor estimado pelo modelo se a distribuição dos resíduos formar figuras geométricas ou caso se acumule em um ponto fora do eixo, a distribuição é tendenciosa. O erro médio relativo e o desvio-padrão da estimativa para cada um dos modelos foram calculados conforme as Eqs. 2 e 3.

$$
\mathrm{P}=\frac{100}{\mathrm{n}} \sum_{\mathrm{i}=1}^{\mathrm{n}} \frac{\left|\mathrm{X}_{\text {exp }}-\mathrm{X}_{\text {pred }}\right|}{\mathrm{X}_{\text {exp }}}
$$

em que:

$\mathrm{P}$ - desvio percentual médio, \%

$\mathrm{X}_{\text {exp }}$ - valores obtidos experimentalmente

$\mathrm{X}_{\text {pred }}$ - valores preditos pelo modelo n - número de dados experimentais

$$
\mathrm{SE}=\sqrt{\frac{\sum_{\mathrm{i}=1}^{\mathrm{n}}\left(\mathrm{X}_{\exp }-\mathrm{X}_{\text {pred }}\right)}{\mathrm{GLR}}}
$$

em que:

SE - desvio padrão da estimativa

$\mathrm{X}_{\exp }$ - valores obtidos experimentalmente

$\mathrm{X}_{\text {pred }}$ - valores preditos pelo modelo

GLR - graus de liberdade do modelo (número de observações menos o número de parâmetros do modelo).

\section{Resultados e Discussão}

As sementes da C. surinamensis submetidas à secagem em secador solar, tinham teor de água inicial de 30,53\% (b.u); após 14 dias atingiram teor de água de equilíbrio $12,28 \%$ enquanto as sementes da C. guianensis tinham teor de água inicial de 43,99\% e após 20 dias atingiram 13,92\% (b.u) (Figura 2).

No interior do secador a temperatura média foi de $33,59^{\circ} \mathrm{C}$ e a umidade relativa média de $51,31 \%$. A secagem das sementes do gênero Carapa ocorreu em taxa decrescente, as maiores taxas de redução de água ocorreram no início da secagem, sendo o maior valor observado para a C. guianensis $0,43 \mathrm{~g} \mathrm{~g}^{-1}$ por dia. No final da secagem a água se encontra fortemente ligada necessitando de maior energia para sua evaporação, resultando em menores valores da taxa de redução de água (Figura 1).

A secagem em menor tempo da C. surinamensis deve possivelmente, ao menor tamanho das sementes (Tabela 2) e maior quantidade de óleo em relação a C. guianensis (Mendonça \& Ferraz, 2007).

Constatou-se que as sementes da C. surinamensis atingiram o equilíbrio no mesmo período relatado pelos extratores de óleo nos estados do Amazonas e Rondônia. Nas usinas as sementes das duas espécies são colocadas para secar em terreiros de concreto com ventilação natural, o ponto ideal de secagem é indicado de modo empírico ao apertar a semente com casca e quebrá-la com facilidade.

O período de produção de sementes de andiroba coincide com o período chuvoso tornando o processo de secagem com ar natural lento. O uso do exaustor no secador solar permitiu que a umidade relativa tivesse pouca variação no período de secagem.

Um dos problemas comumente relatados durante a secagem com ar natural é o elevado teor de umidade final da semente em equilíbrio com a umidade relativa e temperatura durante o período de secagem (Resende et al., 2009). É importante ressaltar o cuidado para evitar fermentação durante o processo visto que o fenômeno de redução do teor de água das sementes envolve simultaneamente a transferência de massa, que pode alterar de forma substancial a qualidade e as propriedades físicas do produto, dependendo do método e das condições de secagem (Medeiros \& Eira, 2006; Elias et al., 2007; Monte et al., 2008).

Estudos semelhantes de secagem natural com espécies oleaginosas apontaram uma redução lenta e demorada do teor 
A. Modelo: Aproximação da difusão
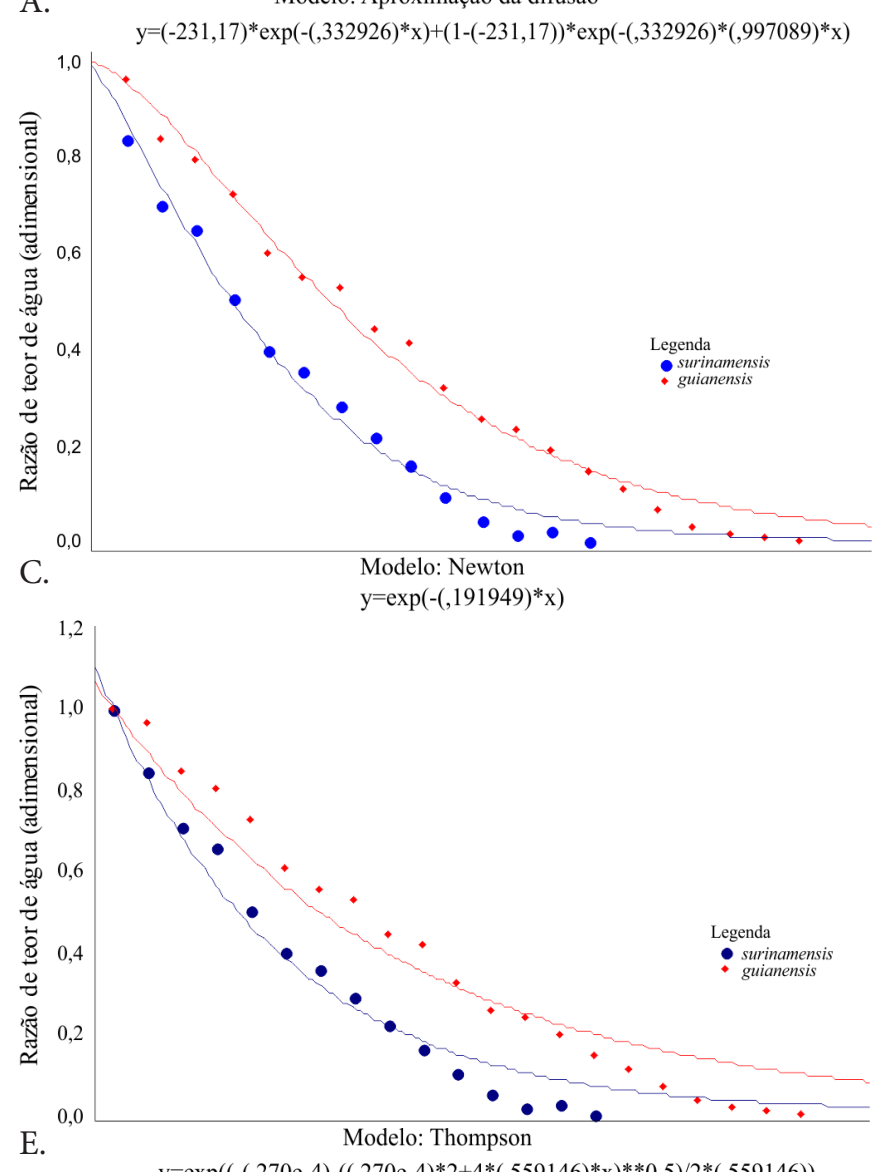

E.

$\mathrm{y}=\exp ((-(, 270 \mathrm{e}-4)-((, 270 \mathrm{e}-4) * 2+4 *(, 559146) * \mathrm{x}) * * 0,5) / 2 *(, 559146))$

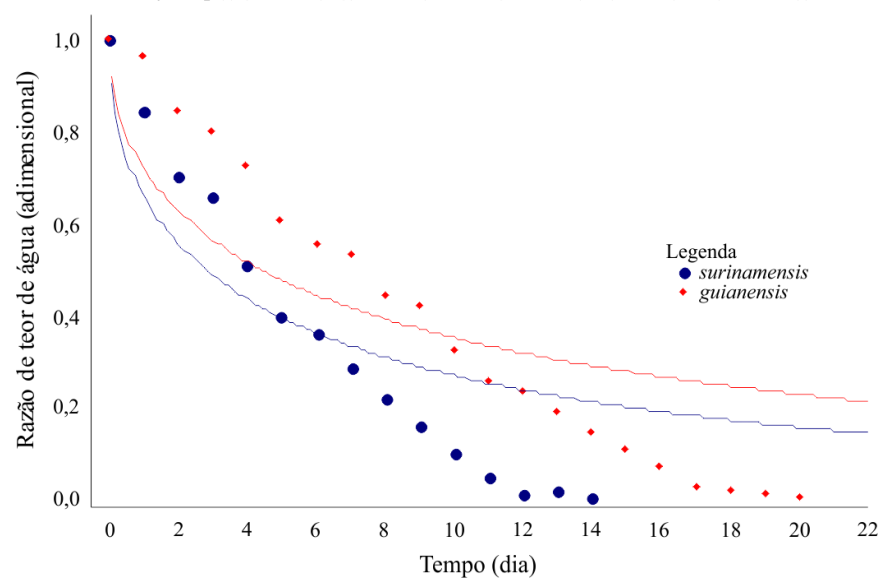

B. Modelo: Logaritmico

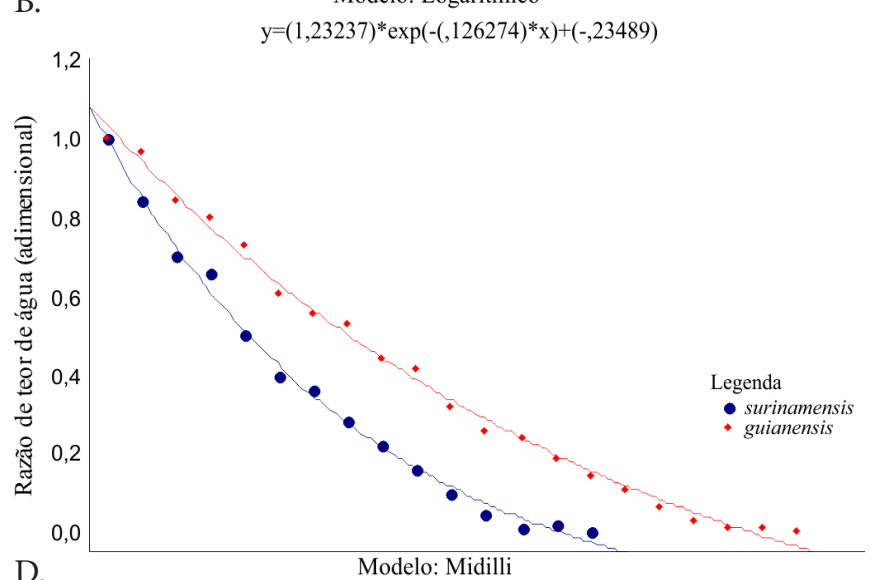

D.
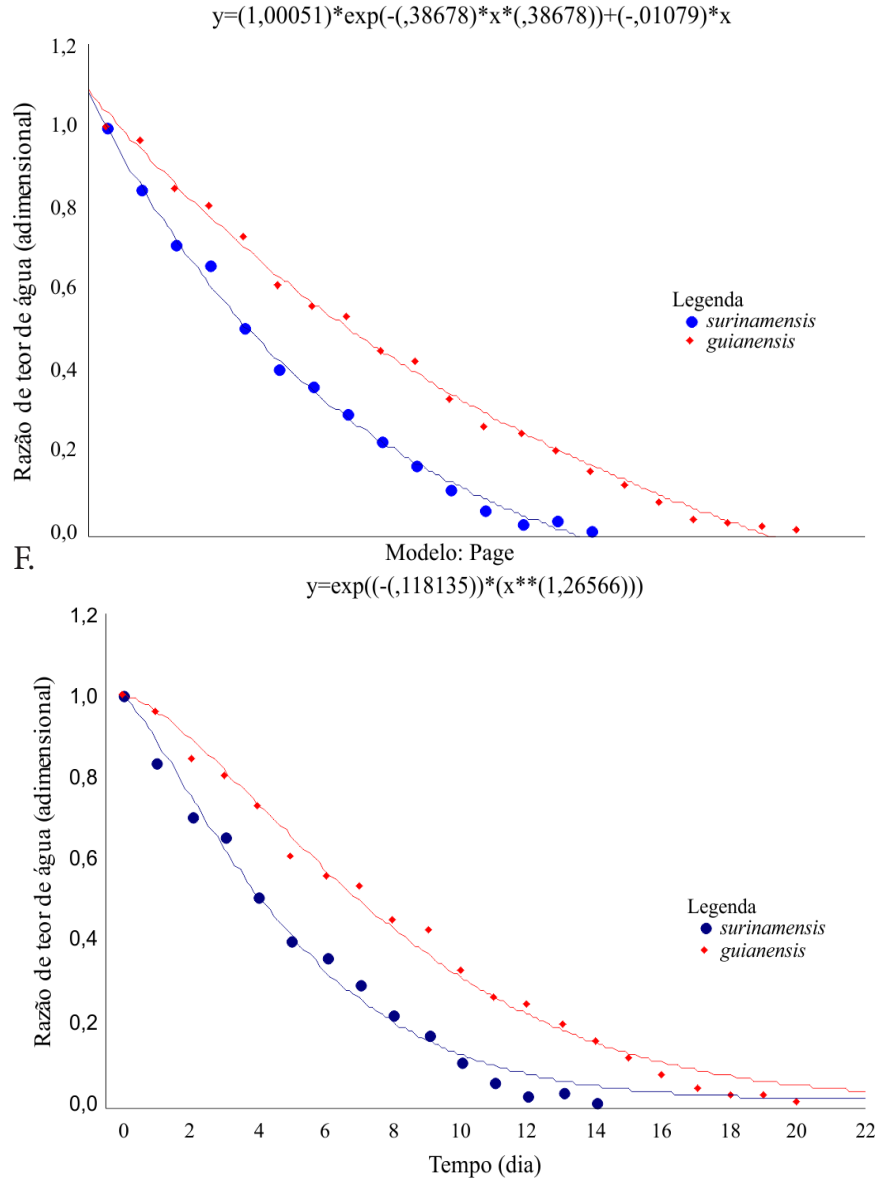

Figura 2. Curvas de secagem das sementes inteiras de andiroba (C. surinamensis Miq e C. guianensis Aubl.) secadas em secador de lona

Tabela 2. Comparação biométrica entre a Carapa surinamensis D.C $(n=150)$ e a Carapa guianensis Aubl. ( $n=150$ ) e número de sementes por quilo, baseado em repetições de $1 \mathrm{~kg}(\mathrm{n}=35)$

\begin{tabular}{lcc}
\hline \multicolumn{1}{c}{ Sementes } & Carapa surinamensis & Carapa guianensis \\
Comprimento & $3,8(2,7-5,5) \mathrm{cm}$ & $4,4(3,1-6,3) \mathrm{cm}$ \\
Largura & $3,1(1,3-4,3) \mathrm{cm}$ & $3,5(2,5-4,6) \mathrm{cm}$ \\
Espessura & $2,8(1,9-3,8) \mathrm{cm}$ & $3,0(1,8-3,9) \mathrm{cm}$ \\
Peso & $16,8(2,2-36,7) \mathrm{g}$ & $27,3(7,1-47,5) \mathrm{g}$ \\
№. sementes $\mathrm{kg}^{-1}$ & $55,3(53-59)$ & $37,2(35-39)$ \\
\hline
\end{tabular}

de água das sementes, independente do ambiente de secagem em relação à secagem artificial. A exemplo das sementes de crambe secadas na planta reduziu o teor de água de 19,9 para $11,6 \%$ após 144 h, mas à sombra sofreram uma redução de 15,8 a 5,6\% após 113 h e em terreiro a redução foi de 15,3 a 7,6\% em 98 h (Oliva, 2010). Sementes de sorgo secadas em terreiro tiveram o conteúdo de água reduzido de 18,9 para 12,58\% após 56 h (Franke et al., 2008).

Em relação aos coeficientes de determinação $\left(\mathrm{R}^{2}\right)$ observouse que somente os modelos Newton e Thompson et al. (1968) apresentaram valores inferiores a $99 \%$ para as duas espécies de andiroba testadas (Tabela 3 ). Salienta-se que apenas o coeficiente de determinação não constitui um bom critério para a seleção de modelos não lineares tornando imprescindíveis avaliações de outros parâmetros (Mamdamba et al., 1996).

Analisando os valores do erro médio relativo $(\mathrm{P})$ concluise que indicam o desvio dos valores observados em relação à curva estimada pelo modelo (Kashaninejad et al., 2007) e 
Tabela 3. Coeficientes de determinação $\left(R^{2}\right)$, desvios percentuais médios $(\mathrm{P})$, desvio-padrão da estimativa (SE) e tendência de distribuição de resíduos para os modelos testados com sementes inteiras de andiroba (C. surinamensis Miq e C. guianensis Aubl.) em secador de lona

\begin{tabular}{lccccc}
\hline \multicolumn{1}{c}{ Modelo } & Carapa & $\mathbf{R}^{\mathbf{2}}$ & $\mathbf{P}$ & $\mathbf{S E}$ & Resíduos \\
Aproximação & surinamensis & 0,9946 & 28,9400 & 0,0012 & aleatória \\
da difusão & guianensis & 0,9941 & 61,6133 & 0,0013 & aleatória \\
\multirow{2}{*}{ Logarítmico } & surinamensis & 0,9980 & 24,0468 & 0,0004 & aleatória \\
& guianensis & 0,9978 & 19,8199 & 0,0004 & aleatória \\
\multirow{2}{*}{ Midilli } & surinamensis & 0,9978 & 25,1919 & 0,0005 & aleatória \\
\multirow{2}{*}{ Newton } & guianensis & 0,9975 & 21,6467 & 0,0005 & aleatória \\
\multirow{2}{*}{ Page (1949) } & surinamensis & 0,9870 & 29,9645 & 0,0025 & tendenciosa \\
& guianensis & 0,9755 & 122,7729 & 0,0050 & tendenciosa \\
\multirow{2}{*}{ Thompson } & gurinamensis & 0,9946 & 18,5924 & 0,0011 & aleatória \\
& guianensis & 0,9955 & 48,4609 & 0,0010 & aleatória \\
& surinamensis & 0,8847 & 37,4606 & 0,0228 & tendenciosa \\
& guianensis & 0,8586 & 268,3614 & 0,0289 & tendenciosa \\
\hline
\end{tabular}

segundo Mohapatra \& Rao (2005) valores menores que 10\% são recomendados para a seleção de modelos indicando, desta forma, uma representação adequada do fenômeno; no entanto, nenhum modelo apresentou valor inferior a $10 \%$. As sementes da C. surinamensis tiveram os menores valores do erro médio relativo (P) no modelo Page (1949) e para a C. guianensis apresentaram menor valor de $\mathrm{P}$ no modelo Logarítmico; já Midilli et al. (2002) apresentaram, em seu modelo, resultados semelhantes para as duas espécies de andiroba (Tabela 3).

O modelo Logarítmico apresentou as menores magnitudes do desvio-padrão da estimativa (SE) para as duas espécies de andiroba seguido pelo Midilli et al. (2002) (Tabela 3). A capacidade de um modelo descrever com fidelidade determinado processo físico, é inversamente proporcional ao valor do desvio-padrão da estimativa (Draper \& Smith, 1998). Desta forma, os modelos matemáticos Logarítmico e Midilli et al. (2002) foram os que melhor se ajustaram aos dados experimentais das sementes de C. surinamensis e $C$. guianensis (Tabela 3).

Os resíduos constituem a diferença entre os valores observados experimentalmente pelos valores estimados pelos modelos, foram plotados em função dos valores estimados de teor de água de equilíbrio. Um modelo é considerado aceitável se os valores residuais se encontrarem próximos à faixa horizontal em torno de zero, não indicando tendenciosidade dos resultados. Se o modelo apresentar distribuição tendenciosa dos seus resíduos, o modelo será considerado inadequado para representar a cinética de secagem (Goneli, 2008). Apenas os modelos Newton e Thompson et al. (1968) apresentaram distribuição tendenciosa dos resíduos para as duas espécies testadas, confirmando que os modelos não se adequam aos dados testados para as duas espécies de andiroba (Tabela 3).

Estudo semelhante realizado em Rondônia em terreiro de concreto para secar café (Coffea canefora Pierre) apontou o modelo Page (1949) como adequado para representação da secagem dos quatro clones testados (Resende et al., 2009).

A constante de secagem $(\mathrm{k})$ pode ser utilizada como aproximação para caracterizar o efeito da temperatura e está relacionada à difusividade efetiva no processo de secagem no período decrescente e à difusão líquida que controla o processo (Babalis \& Belessiotis, 2004). As sementes da C. surinamensis apresentaram os maiores valores da constante (k) e menores valores biométricos comparados a C. guianensis; torna-se possível que isto tenha contribuído para o alcance do teor de água de equilíbrio em menor tempo (Tabela 4).

O parâmetro "n" possui um efeito de moderação do tempo e corrige os prováveis erros resultantes da negligência da resistência interna para a transferência de água (Guedes \& Faria, 2000). O valor de "n" foi maior para as duas espécies no modelo Page (1949) e menor em Midilli et al. (2002). Os parâmetros "a", "b" e "c" tiveram valores semelhantes para as duas espécies de andiroba nos modelos testados (Tabela 4). A superioridade dos modelos Logarítmico e Midilli et al. (2002) em relação aos demais testados pode ser explicada pelo maior número de parâmetros (Tabela 4).

Tabela 4. Parâmetros dos modelos testados com sementes inteiras de andiroba (C. surinamenis Miq e C. guianensis Aubl.) em secador solar

\begin{tabular}{|c|c|c|c|c|c|c|}
\hline \multirow{2}{*}{ Modelos } & \multirow{2}{*}{ Carapa } & \multicolumn{5}{|c|}{ Parâmetros } \\
\hline & & a & b & C & k & $\mathbf{N}$ \\
\hline \multirow{2}{*}{$\begin{array}{l}\text { Aproximação } \\
\text { da difusão }\end{array}$} & surinamensis & $-231,166$ & 0,9970 & & 0,3329 & \\
\hline & guianensis & $-286,834$ & 0,9968 & & 0,2334 & \\
\hline \multirow{2}{*}{ Logarítmico } & surinamensis & 1,2323 & & $-0,2348$ & 0,1262 & \\
\hline & guianensis & 1,4852 & & $-0,4523$ & 0,0636 & \\
\hline \multirow{2}{*}{ Midilli } & surinamensis & 1,0005 & $-0,0107$ & & 0,3867 & 0,3867 \\
\hline & guianensis & 1,0355 & $-0,0119$ & & 0,2870 & 0,2870 \\
\hline \multirow{2}{*}{ Newton } & surinamensis & & & & 0,1919 & \\
\hline & guianensis & & & & 0,1168 & \\
\hline \multirow{2}{*}{ Page (1949) } & surinamensis & & & & 0,1181 & 1,2656 \\
\hline & guianensis & & & & 0,0408 & 1,4644 \\
\hline \multicolumn{2}{|c|}{ Thompson et al. surinamensis } & 0,0000 & 0,5591 & & & \\
\hline (1968) & guianensis & 0,0000 & 0,4805 & & & \\
\hline
\end{tabular}

\section{Conclusões}

1. Os modelos matemáticos logarítmico e Midilli et al. (2002) foram os que melhor se ajustaram aos dados experimentais para as sementes das duas espécies de andiroba.

2. O tempo de secagem para as sementes da Carapa surinamensis foi menor do que para Carapa guianensis.

\section{Literatura Citada}

Almeida, F. de A. C.; Duarte, M. E. M.; Mata, M. E. R. M. C. Tecnologia de armazenamento em sementes. Campina Grande: UFCG, 2006. 402p.

Babalis, S. J.; Belessiotis, V. G. Influence of the drying conditions on the drying constants and moisture diffusivity during the thin-layer drying of figs. Journal of Food Engineering, v.65, p.449-458, 2004. http://dx.doi.org/10.1016/j.jfoodeng.2004.02.005

Brasil. Ministério da Agricultura e Reforma Agrária. Secretaria Nacional de defesa Agropecuária. Regras para análise de sementes. Brasília: MARA. 2009. 365p.

Draper, N. R.; Smith, H. Applied regression analysis. 3. ed. New York: John Wiley \& Sons, 1998. 712p. http://dx.doi. org/10.1002/9781118625590

Elias, M. C.; Guerra, R. A.; Elias, S. A. A.; Antunes, P. L. Operações de pré-armazenamento, armazenamento e conservação de grãos. In: Elias, M.C (ed.) Pós-colheita de arroz: Secagem, armazenamento e qualidade. Pelotas: Edgraf UFPel, 2007. p.147-244. 
Franke, L. B.; Torres, M. A. P.; Lopes, R. R. Performance of different drying methods and their effects on the physiological quality of grain sorghum seeds (S. bicolor (L.) Moench). Revista Brasileira de Sementes, v.30, p.177-184, 2008. http://dx.doi.org/10.1590/ S0101-31222008000300024

Guedes, A. M. M.; Faria, L. J. G. Determinação da constante de secagem de urucum (Bixa orellana L.) em secador convectivo de leito fixo. Revista Brasileira de Produtos Agroindustriais, v.2, p.73-86, 2000.

Goneli, A. L. D. Variação das propriedades físicas-mecânicas e da qualidade da mamona (Ricinus communis L.) durante a secagem e o armazenamento. 2008. 186p. Viçosa: UFV. Tese Doutorado

Henderson, S. M.; Pabis, S. Grain drying theory: Temperature effect on drying kinetics. Journal of Food Engineering, v.39, p.37-44. 1961.

Kashaninejad, M.; Mortazavi, A.; Safekordi, A.; Tabil, L. G. Thinlayer drying characteristics and modeling of pistachio nuts. Journal of Food Engineering, v.78, p.98-108, 2007. http://dx.doi. org/10.1016/j.jfoodeng.2005.09.007

Kenfack, D. Resurrection in Carapa (Meliaceae): A reassessment of morphological variation and species boundaries using multivariate methods in a phylogenetic context. Botanical Journal of Linnean Society, v.165, p.86-221. 2011. http://dx.doi. org/10.1111/j.1095-8339.2010.01104.x

Medeiros, A. C. S.; Eira, M. T. S. Comportamento fisiológico, secagem e armazenamento de sementes florestais nativas. Colombo: EMBRAPA. 2006. 13p.

Mendonça, A. P.; Ferraz, I. D. K. Óleo de andiroba: processo tradicional da extração, uso e aspectos sociais no estado do Amazonas, Brasil. Acta Amazonica, v.37, p.353-364, 2007. http:// dx.doi.org/10.1590/S0044-59672007000300006

Midilli, A.; Kucuk, H.; Yapar, Z. A new model for single layer drying. Drying Tecnology, v.20, p.1503-1513, 2002. http://dx.doi. org/10.1081/DRT-120005864
Mohapatra, D.; Rao, P. S. A thin layer drying model of parboiled wheat. Journal of Food Engineering, v.66, p.513-518, 2005. http://dx.doi. org/10.1016/j.jfoodeng.2004.04.023

Monte, J. E. C.; Martins, J. H.; Lopes, D. C.; Monteiro, P. M. B.; Pinto, P. R. Sistema automático para secagem de produtos agrícolas em camada fina. Acta Scientiarum. Agronomy, v.30, p.307-312, 2008.

Oliva, A. C. E. Qualidade de sementes de crambre submetidas a métodos de secagem e períodos de armazenamento. São Paulo: UNESP. 2010. 87p. Dissertação Mestrado

Page, G. E. Factors influencing the maximum rates of air drying shelled corn in thin layers. West Lafayette: Purdue University, 1949. s.p. M.S. Dissertation

Resende, O.; Arcanjo, R. V.; Siqueira, V. C.; Rodrigues, S. Modelagem matemática para a secagem de clones de café (Coffea canephora Pierre) em terreiro de concreto. Acta Scientiarum Agronomy, v.31, p.189-196, 2009. http://dx.doi.org/10.4025/actasciagron. v31i 2.588

Resende, O.; Arcanjo, R. V..; Siqueira, V. C,; Rodrigues, S.; Kester, A. N.; Lima de, P. P. Influência do tipo de pavimento na secagem de clones de café (Coffea canephora Pierre) em terreiros de concreto e chão batido. Revista Brasileira de Produtos Agroindustriais, v.9, p.171-178, 2007.

Silva, O. R .R. F.; Nóbrega, M. B. M.; Gondim, T. M. S. Cultivo da mamona. Embrapa Algodão. 2007. http://mamona.agriculture. com.br/colheita.php.

Thompson, T. L.; Peart, R. M.; Foster, G. H. Mathematical simulation of corn drying: A new model. Transactions of ASAE, v. 11, p.582586, 1968. http://dx.doi.org/10.13031/2013.39473

Wang, C. Y.; Sing, R. P. Use of variable equilibrium moisture contente in modeling rice drying. Transaction of ASAE, v.11, p.668-672. 1978. 